Meta

Journal des traducteurs

Translators' Journal

\title{
Index des mots et sujets dans le volume 22
}

Volume 22, numéro 4, décembre 1977

URI : https://id.erudit.org/iderudit/003207ar

DOI : https://doi.org/10.7202/003207ar

Aller au sommaire du numéro

Éditeur(s)

Les Presses de l'Université de Montréal

ISSN

0026-0452 (imprimé)

1492-1421 (numérique)

Découvrir la revue

Citer ce document

(1977). Index des mots et sujets dans le volume 22. Meta, 22(4), 303-307.

https://doi.org/10.7202/003207ar d'utilisation que vous pouvez consulter en ligne.

https://apropos.erudit.org/fr/usagers/politique-dutilisation/ 


\section{INDEX DES MOTS ET SUJETS TRAITÉS DANS LE VOLUME 22}

Acadie, 4, p. 292.

Acculturation, 1, p. 69 ; 3, p. 232.

Action commerciale, 3, p. 212.

Activité terminologique des entreprises, 1, p. 51.

Actualité terminologique, 1, p. 50.

Adéquation, 4, p. 271.

Adjectif, 2, p. 150

Administration de la justice, 1, p. 16.

Adresses des organismes de terminologie, 2, p. 132 .

Affecter, 2, p. 145, 152

Alignement, 2 , p. 145 .

Allumé, 2, p. 134.

ALPAC (rapport), 1, p. 55.

Amiral des forces aéronavales, 4, p. 262.

Analogie, 4, p. 280.

Analyse des tâches du terminologue, 1, p. 51.

An, 4, p. 280

Année, 4, p. 280.

Année-homme, 4, p. 274

Annonces publicitaires, 1, p. 70 .

Anonymat, 1, p. 85 .

Apprébiension du réel, 4, p. 254.

Argot, 4, p. 253.

Argumentaire, 2, p. 145.

Arrondi, 2, p. 135 .

Art oratoire des Indiens, 1, p. 13.

Assemblée délibérante, $4, \mathrm{p} .275$.

Association des traducteurs littéraires, 1, p. $43,83$.

Association française de normalisation, $2, \mathrm{p}$. 129.

Association française de terminologie, 2, p. 128.

Associations professionnelles, 4, p. 298.

Association technologique de langue française d'Ottawa, 1, p. 46.

Assurances, 1, p. 98.

Atmosphère d'intimité, 3, p. 183.

Attitude d'écoute, 4, p. 295.

Auxiliaire d'aspect, 2, p. 150.

Auxiliaire de temps, 2, 150 .

Auxiliaire de voix, 2, p. 150.

Avoir du vent dans les voiles, 2, p. 135.

Banque de terminologie, 3, p. 184.

Banque de terminologie de l'Université de Montréal, 1, p. 50

Benoit, Georges, 1, p. 59.

Bible (traduction de la), 3, p. 192

Bibliographie de la traduction, 1, p. 72.

Bibliographie sélective, 3, p. 227 .

Bien, 3, p. 208.

Biennale de la langue française, 4, p. 292.

Bilan (réponse en), 3 , p. 188.

Bilinguisme, 1, p. $19 ; 3$, p. 232 ; 4, p. 284.

Bilinguisme de coexistence, 4, p. 292 .

Bilinguisme fédéral, 1, p. 45.

Boulou, 3, p. 192.

Brindezingue, 2, p. 135.
Brouillard (être dans le), 2, p. 135.

Bruit, 3, 187.

Brûlé, Etienne, 1, p. 7.

Bruyères, John des, 1, p. 17.

Bulletins de linguistique (Académie canadienne-française), 1, p. 48 .

Bulletins de terminologie, 1, p. 46

Bureau des traductions, 1, p. 57.

Bureau fédéral des traductions, 4, p. 293.

Cajeux, 1, p. 19.

Canasson, 2, p. 111.

«Capaciteur $», 3$, p. 219.

Capitaux fébriles, 2, p. 145 .

Caribou, 4, p. 280.

Catégorie grammaticales, 2, p. 148.

Cautère, 4 , p. 281.

Centre de terminologie, 1, p. $47 ; 2$, p. 127.

Charte internationale des traducteurs, 3, p. 234.

Cheval, 2, p. 111.

Cheval-vapeur, 3, p. 172.

Civilisation et vocabulaire, 3 , p. 194.

Clignotant, 2, p. 145.

Cloueur, 4, p. 260.

Cloueuse, 4, p. 260.

Code Morin, 4, p. 276

Colloque des terminologues du Québec, 1, p. 98.

Colonisation et traduction, 4, p. 253.

Coma (dans le), 2, p. 135.

Combiné portatif, 4 , p. 272

Comité de linguistique (Radio-Canada), 1, p. 48.

Comité de terminologie française (Ordre des comptables), 1, p. 49 .

Comité interbancaire de terminologie, 2, p. 164.

Comité interentreprise, 1, p. 99.

Comité international de terminologie scientifique et technique, 1, p. 53 .

Comités consultatifs canadiens de I'ISA, 1, p. 53 .

Commerce, 1, p. 16.

Commercialisation, 3, p. 211.

Commission des Communautés européennes, 2 , p. 129 .

Commission de terminologie, 1, p. 51.

Communication, 4, p. 284.

Communication juridique, 4 , p. 289.

Comptabilisation à la valeur de consolidation, 4, p. 282.

Comptabilité, 4, p. 281, 282.

Compte sur livret, 2, p. 145.

Concours de recrutement, 2, p. 158.

Condensateur, 3, p. 220.

Condition des traducteurs, 4, p. 296.

Condition de travail des traducteurs, 4, p. 299.

Congés annuels, 4, p. 293.

Congés compensateurs, 4, p. 293.

Congrès de la F.I.T., 1, p. $93 ; 3$, p. 230. 
Congrès international de linguistique appliquée, 2, p. 166.

Conquête des marchés, 3, p. 212.

Conseil des arts de 1'Ontario, 1, p. 82

Conseil des traducteurs et interprètes du $\mathrm{Ca}$ nada, 1, p. 86

Conseil international de la langue française, 2 , p. 129 .

onstatifs, 4 , p. 288.

Convenablement, 3, p. 209.

Convention universelle sur le droit d'auteur, 1, p. 80.

Correctement, 3, p. 205.

Cotation des épreuves, 2, p. 160.

Coup de téléphone, 3, p. 183.

Cours de rédaction, 1, p. 97.

Cramahé, H. T., 1, p. 17.

Création néologique, 3, p. 233.

Créativité spontanée, 4, p. 294.

Crémaillère, 3 , p. 172.

Critères de sélection, 4, p. 272.

Critères des recours à la traduction, 1, p. 196.

Cugnet, François-Joseph, 1, p. 21.

Cuit, 2, p. 135.

Culbuté, 2, p. 135.

Culture, 2, p. 148 .

Culture française, 4, p. 292.

Datations, 4 , p. 280

Datum, 1, p. 53

Décisions exécutoires, 4, p. 288.

Décodage, 4, p. 278.

Démarche du terminologue, 3, p. 186

Déséquilibre, 2 , p. 145 .

Développement des langues nationales, $3, p$ 231.

Dialectes, 4, p. 253, 256

Dictionnaire de la langue huronne, 1, p. 7.

Dictionnaire économique et financier, 2 , p. 145.

Dictionnaire militaire, 1, p. 46 .

Dictionnaire normatif, 4, p. 280.

Didactique des langues, 2, p. 146

Différends sur la qualité des traductions, 4 p. 298.

Difficultés de recouvrement, 3, p. 183 .

Diffusion des travaux de terminologie, $1, p$. 99.

Dimensions transformatrices de la traduction, 4, p. 252.

Directeur artistique, 2, p. 126

Directeur de scène, 2 , p. 126 .

Discours, 2, p. 110.

Division de la recherche terminologique (Ottawa), 1, p. 50 .

Documentation, 1, p. $99 ; 2,114$.

Dont acte, 4, p. 289

Doublage des films, 1 , p. 81 .

Double opinion, 3, p. 196.

Droit, 4, p. 287.

Droit d'auteur, 1, p 80 ; 4, p 299.

Droit de regard du traducteur, 1, p 83.

Droits de 1'homme, 2, p. 143.

Echange de personne à personne, 3, p. 183.

Ecriture des unités, 4, 274.

Editeur et traduction, 1, p. 82

Effectifs en terminologie, 1, p. 52.

Electrotechnique, 3, p. 218.

Eméché, 2, 134.
Emetteur-récepteur portatif, 4, p. 271.

Emprunt, 4, 294.

Enoncé d'opinion du rapport comptable, 3, p. 195.

Enseignement alterné, 1, p. 97.

Enseignement de la terminologie, 1, p. 52.

Enseignement des langues, 2, p. 146.

Equitablement, 3, p. 205.

Equivalences, 3, p. 192.

Etapes de recherche ponctuelle, 4, p. 258.

Etat voisin (dans un), 2, p. 134.

Ethique de travail, 3, p. 183.

Etre paqueté, 2, p. 136.

Etre parti pour la gloire, 2, p. 136.

Etre sur une baloune, 2, p. 136.

Etui, 4, p. 259.

-euse, 4, p. 260

Exactitude, 3, p. 199

Expert-comptable, 3, p. 197.

Expressions consacrées, 2, p. 113.

Extension juridique, 4, p. 289.

Extension sémantique, 3, p. 234.

« Facturation progressive 》, 4, p. 282.

Facturation proportionnelle, 4, p. 282.

Farci, 2, p. 135 .

Fédération internationale des traducteurs, 1, p. 86.

Fiches terminologiques, 1, p. 52.

Fichiers individuels, 1, p. 46.

Fidèle, 3, p. 201.

Fidèlement, 3, p. 207.

Fidélité, 4, p. 252.

Fidélité lexicale, 1, p. 98.

Fidélité stylistique, 1, p. 98.

Fièvre de Bercy, 2, p. 135.

Finalités sociales de la traduction, 4, p. 257.

Formation des traducteurs, 4, p. 299.

Fonctions d'un service de traduction, 1, p. 97.

Fonction performative du discours, 4, p. 286.

Formation du traducteur, 1, p. 97.

Football, 3, 227

Francisation, 1, p. $96 ; 4$, p. 283.

Francisation (tendance à la), 1, p. 19

Franco-canadien, 4, p. 290.

Francophonie canadienne, 4, p. 291.

Franglais, 4, p. 255

Frécop, 4, p. 270.

Fréquence, 4, p. 271.

Gai, 2, p. 134.

Garçon de café, 3, p. 173.

Garde du corps, 3, p. 172.

Garneau, François-Xavier, 1, p. 31, 33.

Gavé, 2, p. 135.

Gelé, 2, p. 135.

Géologie, 4, p. 290.

Gestion commerciale, 3, p. 212.

Gérin-Lajoie, Antoine, 1, p. 29

Gestion marketing, 3, p. 214.

Gestion théâtrale, 2, p. 125.

Givre, 2, p. 135.

Glissements sémantiques, 4, p. 294.

Gris, 2, p. 134, 135 .

Gugy, Conrad, 1, p. 18.

Haut Comité de la langue française, 2, p. 128.

Heure d'arrivée, 4, p. 293

Heures-hommes, 4, p. 272 
Historique des professions, 1, p. 68.

Horaire variable, 4, p. 293.

Houille, 4, p. 280.

Humecté, 2, p. 135.

Hydro-Québec, 1, p. 49.

Impact du message, 4, p. 294.

Implications linguistiques de la loi sur la langue officielle, 1, p. 93.

Incitations, 2, p. 145 .

Informatique, 2, p. 153.

Infoterm, 2, p. 130

Intermédiaire-terminologue, 3, p. 189.

Interphone portatif, 4, p. 271.

Interprétation, 2, p. 166.

Interprétation (historique de 1'), 1, p. 5.

Interprètes, 4, p. 293.

Interprètes judiciaires, 1, p. 11.

Interprètes militaires, 1, p. 11 .

Interprètes-résidents, 1 , p. 6

Intraduisibilité, 3, p. 233 .

Inventaire des traductions, 1 , p. 96

Inventaire des travaux de terminologie, $1, \mathrm{p}$. 52.

a Items $», 4$, p. 277.

Ivre, 2, p. 132 .

Journalisme bilingue, 1, p. 21.

Journée de travail, 4, p. 293.

Jugement de la qualité, 1, p. 84.

JURIVOC, 1, p. 53.

Langage des psychotiques, 4, p. 285.

Langage juridique, 4 , p. 288.

Langue, 4 , p. 256 .

Langue de communication officielle, 1, p. 94.

Langue de spécialités, 1, p. 94.

Langue et identité culturelle, 4, p. 292.

Langage et opacité, 4, p. 293.

Langue et traduction, 4, p. 284.

Langue française, 4, p. 292.

Langue institutionnalisée, 1, p. 94

Laugue maternelle et traduction, 3, p. 230 ; 4,300 .

Langue québécoise, 3, p. 228.

Langues de faible diffusion, 3, p. 231.

Langues étrangères, 1, p. 64 .

Langues indiennes, $1, \mathrm{p} .11$.

Lecteurs-réviseurs, $1, \mathbf{p} .83$.

Lexiques bilingues, 1, p. 47.

Linguistique computationnelle, 3, p. 184.

Linguistique et traduction, 2, p. 152 .

Littéralité, 1, p. 25.

Loi sur la langue officielle, 1, p. 93.

Loyalement, 3, p. 205.

Machabé, 2, p. 135

Maniabilité, 4, p. 271.

Marchandisage, 3, p. 216.

\& Marchandises sèches \$, 1, p. 19.

Marchéage, 3, p. 211.

Marché de la traduction, 1, p. 79.

Marketing, 3, p. 211.

Marques linguistiques, 4, p. 288.

Maturin, Gabriel, 1, p. 17.

Mercantique, 3, p. 212

Mercatique, 3, p. 211.

Méthode de la mise en équivalence, 4, p. 282.

Méthodologie de la terminologie, 1 , p. 95.

Méthodologie du travail en comite, 1, p. 99.
Metteur en scène, 2, p. 126

Mise en marché, 3, p. 211.

Mission terminologie, 1, p. 51 .

Mobilophone, 4, p. 270.

Mode d'entrée des données, 3, p. 184.

Modèles culturels, 4, p. 255.

Mot grammatical, 2, p. 112.

Motivation, 4, p. 271.

Mot sémantique, 2, p. 112.

Mot-terme, 2, p. 110.

Mouillé, 2, p. 135

Murdingué, 2, p. 135.

Négoce, 3, p. 212.

Négocialogie, 3, p. 212.

Négociologie, 3, p. 212.

Néologie, 1 , p. $52 ; 2$, p. 115.

Nicolet, Jean, 1 , p. 8.

Niveaux linguistiques, 4, p. 288.

Noir, 2, p. 134, 135.

Nom commun, 2, p 149

Normalisation, 1, p $52 ; 3$, p. 234 .

Normalisation du vocabulaire, 1, p. 51.

Normalisation terminologique, 1, p. 95.

Norme de pronenciation, 4 , p. 281.

Normes de qualités en traduction, 1, p. 96.

Notion, 2, p. 112.

Objectivement, 3, p. 206.

Office de la langue française, 1, p. 48.

Options et servitudes, 4, p. 245

Organisation internationale de normalisation (ISO), 2 , p. 130.

Organisation professionnelle, 4, p. 298

Organismes de terminologie, 2, p. 126.

Ordre du jour, 4, p. 274.

Ostensif, 4, p. 260.

Ours (soûl comme un), 2, p. 136.

Ouvrages savants, $1, \mathrm{p} .82$.

Ouvrages scolaires, 1, p. 82

Paf, 2, p. 134.

Palan pneumatique à piston, 2, p. 112.

Paqueter (se) la fraise, 2, p. 136.

Paroisse de Saint-Jean-le-Rond, 2, p. 135.

Partage des tâches en terminologie, 1, p. 95 .

Partenaires commerciaux, 2, p. 145.

Participe passé, 2, p. 150.

Parure de noce, 3, p. 221.

Pause-repas, 4, p. 293

Peau d'orange, 3, p. 172.

Perfectionnement linguistique, 4, p. 284.

Performatifs, 4, p. 288.

Petit Robert, 4, p. 278.

Placards (traduction des), 1, p. 16.

Piastre, 1, p. 19.

Place de la traduction au Canada, 1, p. 66.

Plan de carrière du traducteur, 1, p. 97.

Planification, 1, p. 62.

Plein, 2, p. 135.

Pluralisme de la francophonie, 4, p. 292.

Point d'exclamation, 3, p. 183.

Politique de francisation, 1, p. 45.

Polonais (soûl comme un), 2 , p. 136.

Pomme de terre, 3, p. 172.

Pompette, 2, p. 134.

Pratique de la traduction, 3, p. 228.

Premiers traducteurs, $1, \mathbf{p}$. 15 .

Prendre une brosse, 2, p. 136.

Prévention des incendies, 1, p. 50. 
Proclamations officielles, 1,p. 16.

Producteur, 2, p 126.

Production en traduction, $1, p, 68$.

Prononciation, 4, p. 281

Protection juridique des traducteurs, 4. 296.

« Puissance » du Canada, 1, p. 31.

Qualité de la traduction, 1 ; p. 84 ; 3, p. 234 ; 4, p. 254.

Qualité de la vie au travail, 1, p. 64

Québécismes, 4, p. 280.

Questions d'affaires, 3, p. 183.

Radiotéléphone portatif, 4, p. 271.

Recherche ponctuelle, 4, p. 258.

Recherche terminologique et traduction, 3, p. 233.

Recherche thématique, 4, p. 258.

Recrutement, 1, p. 60 .

Rédaction, 1, p. 97

Régie de la langue française, 2, p. 127.

Régionalismes, 4 , p. 280 .

Régisseur adjoint, 2, p. 126

Régisseur général, 2, p. 126

Régularité, 3, p. 199

Régulièrement, 3, p. 206

Remplissage, 3, p. 218 .

Rémunération des traducteurs, 1, p. 60 .

Rencontre R.L.F.-S.T.Q., 1; p. 93 ,

Répertoire bibliographique de la traduction, 2, p. 151 .

Réponses d'une banque de terminologie, 3, p. 187.

Réponses pertinentes, 3, p. 187

Rescapé, 4, p. 280.

Réseau des organismes de terminologie, 2, p. 131.

Résistance, 3, p. 220

Résisteur, 3, p. 219

Retour d'information, 2, p. 145.

REZO, 1, p. 56.

Rivière de'Saint-Laurent, 1 , p. 19.

Robe de chambre, 3 , p. 172:

Robichaud, Damien-T., 1, p. 59.

Rôle de la traduction, 4 , p. 253.

Rốle du langage, 4, p. 294.

Rond, 2, p. 135.

Rondibé, 2, p. 135.

Rosse, 2, p. 111 .

Saint Jérôme, 1, p. 88.

Schizolinguistique, $4, p, 287$

Sciences de la terre, 4, p. 290

Sciences occultes; $2 ;$ p. 153 .

Secrétaires bilingues, 1, p. 15

Sectionné, 4, p. 259 .

Sections professionnelles, 3, p. 237.

Semaine de travail, 4 , p. 293.

Service de linguistique de Radio-Canada, 2, p. 128.

Services de traduction dans les entreprises, 1, p. 49.

Sexologie, $3 ; \mathrm{p} .227$

SI (système international d'Unités), 4, p. 272.

Signature des traductions, 1, p. 23,85 .

Signes diacritiques, 4, p. 291 .

Signes linguistiques, 4 , p. 288

Silence, 3 , p. 187

Silence et communication, 4, p. 295.
Sincère, 3, p: 201

Sincèrement, 3 , p. 206.

Sincérité, 3, p. 199, 201.

Situation du françails au Canada, 3, p. 234.

Situation fiscale du traducteur, 4, p. 299.

Situation juridique des traducteurs, 4, p. 297.

Situation sociale du traducteur, 4, p. 299

Société des traducteurs du Québec, 3, p. 236.

Sociologie du traduire; 4, p. 254.

Soûl, 2, p. 133.

Spécialiste et traducteur, 4, p. 295.

Spécialistes, 4, p. 259.

Spécialisation, 3, p. 230

Stanley House, 1, p. 49.

Structure des fichiers, 3, p. 185.

Styles, 2, p. 104.

Stylistique comparée, 4, p. 243

Subventions à la traduction, 1, p. 79 .

Superenfer, 2, p. 145.

Synecdoque et traduction, 4, p. 284.

Syntoniser, 3, p. 219.

Table à café, 2, p. 138.

Table à cocktail, 2, p. 138.

Table à fumer, 2 , p. 142 .

Table à lampe, 2, p. 141.

Table apéritif, 2 , p. 139

Table a thé, 2, p. -139 .

Table auxiliaire, $2, \mathrm{p}$. 139

Table basse, 2 ; p. 138 .

Table buffet, 2 , p. 139

Table d'appoint, 2 , p. 139

«Table de bout $\gg, 2$, p. 140

Table d'éclairage, $2, \mathrm{p} .141$.

Tables de salon, 2, p. 137.

Table de séjour, 2; p. 139 .

Table fumeur, 2, p. 142.

Table luminaire, 2 , p. 141.

Table tabac, 2, p. 142 .

Tabous linguistiques, 4 , p. 280

TAUM, 1, p. 56 .

Tantièmes, 1, p. 80.

Tarif de la traduction littéraire, 1, p. 79

Tarif-taux, 1, p. 19.

Technique généale, 3 , p. 226

Télécommunications, 4, p. 290 .

Terme, 2, p. 113.

Terme-mot, 2, p. 110.

Termes de (en), 2, p. 145

Termes réels (en), 2, p. 145

Terminologie, $4, p_{2} 291,300$.

Terminologies, 3 , p. 184 .

Terminologie comparée, 4, p. 258.

Terminologie comptable, $3, p, 195$.

Terminologie des industries dù papier, 1, p. 49.

Terminologie et documentation, 1, p. 62

Terminologie (histoire de la), 1, p. 45.

Terminologue et banque de terminologie, 3 , p. 185.

Terminologue et mot, 2, p. 112.

Terminologue et spécialiste; 4, p. 296.

Tête de pont, 3, p. 172.

Tête d'injection, $3, \mathrm{p} .172$

Textes dramatiques, 4, p. 256.

Textes informatifs, 1, p. 67

Théorie de la terminologie, 1, p. 52

Thériaque, 4 , p. 261.

Tírage des traductions; $1 ;$ p: 80 . 
Top-toc, 4, p. 271.

Tourtière, 4, p. 280

Traducteur, 4, p. 297.

Traducteur autonome, 1, p. 98.

Traducteur dans l'entreprise, 1, p. 97.

Traduction de la poésie, 4, p. 255.

Traducteur et banque de terminologie, $3, p$. 186.

Traducteur et juriste, 4, p. 294.

Traduction au Canada, 3, p. 232.

Traduction automatique (histoire de la), 1, p. 54.

Traduction électronique, 1, p. 64

Traduction esthétique, 1, p. 67.

Traduction et acculturation, 1, p. 69.

Traduction médicale, 4, p. 295.

Traduction (place de la langue en), 4, p. 284

Traduction publicitaire, 1, p. 70 .

《Traîne 》, 1, p. 19.

Trait d'union avec anti-, 4, p. 281.

Traitements, 4, p. 293.

Translatique, 1, p. 92 .

Translitération, 4, p. 291.

Transmission des cultures, 4, p. 294.

Transparence, 4, p. 252, 294.

Transports, 4, p. 290.
Troncature non sélective, 3, p. 189.

Tronc commun, 1, p. $95 ; 4$, p. 282.

Uniformisation de l'orthographe, 4, p. 281.

Unités de mesure, 4, p. 272.

Unité de traduction, 3, p. 234

Unité terminologique, 2, p. 113.

Univers de discours juridique, 4, p. 289.

Université de Saskatchewan, 1, p. 55.

Usagers d'une banque de terminologie, 3, p. 185.

Utilisateur, 4, p. 297.

Vaisseau de guerre, 3, p. 172

Valeur communicative, 2, p. 148.

Vignes du Seigneur (être dans les), 2, p. 134.

Vocabs (CN), 1, p. 49 .

Vocabulaire de l'éducation, 1, p. 50.

Vocabulaire et civilisation, 3, p. 194.

Vocabulaire franco-iroquois, 1, p. 6

Vocabulaire général d'Hector Carbonneau, 1, p. 46.

Vocabulaire nucléaire, 1, p. 49

Vocabulaires spécifiques, 1, p. 95.

Voltampère, 4, p. 273.

Wigwams, 1, p. 10. 